\title{
Olaf Nohr
}

\section{Vernunft als Therapie und Krankheit: Medizinische Denkfiguren in der Geschichte der Philosophie}

In:

Philip van der Eijk / Detlev Ganten / Roman Marek (Hrsg.): Was ist Gesundheit? :

Interdisziplinäre Perspektiven aus Medizin, Geschichte und Kultur

ISBN: 978-3-11-071333-6. - Berlin / Boston: De Gruyter, 2021

(Humanprojekt : Interdisziplinäre Anthropologie ; 18)

Teil 1: Antike Grundlagen und philosophische Gesundheitsbegriffe

S. $15-29$ 


\title{
Olaf Nohr
}

\section{Vernunft als Therapie und Krankheit: Medizinische Denkfiguren in der Geschichte der Philosophie}

\begin{abstract}
Reason as Therapy and Illness: Medical Figures of Thought in the History of Philosophy. This paper tackles the question how philosophers have used medical metaphors, analogies or aspects of medical theories in their works. It discusses the idea of ancient Greek philosophy as a medicine of the soul, as well as the Christian surgery of the text-body and finally, how madness became a central problem for the philosophical conception of reason.
\end{abstract}

Interdisziplinäres Denken ist keine Erfindung der Neuzeit: Seit mehr als 2000 Jahren üben medizinische Denkfiguren einen erheblichen Einfluss auf die philosophische Theoriebildung aus. Als „medizinische Denkfigur“ werden hier Theoriebausteine aus der Medizin und Heilkunde bezeichnet, die - oftmals als Metapher oder Analogie - für philosophische Problemlösungen fruchtbar gemacht wurden. Der vorliegende Beitrag möchte die wichtigsten Stationen dieses Austausches im Zeitraffer nachzeichnen. ${ }^{1}$ Betrachtet man die Geschichte der medizinischen Denkfiguren von den antiken Therapien der Seele über die christliche Chirurgie des Textkörpers bis zu den Diskussionen um die Unterscheidung von Vernunft und Wahnsinn, so wird deutlich, dass sich die philosophischen Vernunftkonzepte stets in einer stillschweigend vorausgesetzten Teilhabe an der Gesundheit artikulieren. Diese Assoziationen von Vernunft und Gesundheit wurde erst erschüttert, als das Phänomen des Wahnsinns im 18. Jahrhundert zu einem zentralen Problem der Vernunftkonstruktion avancierte und zum ersten Mal der Gedanke einer kranken Vernunft aufkam. Doch wo liegen die Wurzeln dieser engen Verbindung von Philosophie, Vernunft und Gesundheit?

1 Zur ausführlicheren Darstellung der folgenden Gedanken: (Nohr 2015)

Olaf Nohr, http://olafnohr.com/

Ә OpenAccess. (c) 2021 Olaf Nohr, publiziert von De Gruyter. (cc) BY-NC-SA Dieses Werk ist lizenziert unter einer Creative Commons Namensnennung - Nicht kommerziell - Weitergabe unter gleichen Bedingungen 4.0 International Lizenz. https://doi.org/10.1515/9783110713336-003 


\section{Antike Ethik als Therapie der Seele}

Im homerischen Epos Ilias wird ein erstes Verständnis von Gesundheit entworfen, das den Menschen als Summe verschiedener Organe und Körperteile konzipiert. Jeder dieser Körperteile hat eine eigene Seele und damit die Macht, den gesamten Menschen in Aufruhr zu versetzen (Homer 2002). In der Odyssee wird dieses Bild um die Figur des „listigen Menschen“ Odysseus erweitert, der seinen Matrosen die Ohren mit Wachs verschließt, um ihre körperlichen Wahrnehmungen vollständig zu unterdrücken und sich selbst am Mast festbindet, um den affektiven Verhaltensautomatismus der körperlichen Regungen zu unterdrücken. Damit wird Odysseus zum ersten Protagonisten einer Geschichte, die sich als „Entdeckung des Geistes“ bei gleichzeitiger „Verdeckung des Körpers“ bezeichnen lässt (Schmitz 1965, S. 366).

Das Ideal des Unterdrückens körperlicher Regungen gibt auch einen Hinweis darauf, weshalb den Zeitgenossen von Hippokrates ca. 400 Jahre später ausgerechnet die Epilepsie als „heilige Krankheit“ galt. Der vollständige Kontrollverlust bei einem epileptischen Anfall steht prototypisch für den Kontrollverlust der Vernunft über den Körper und setzt der Selbstermächtigung des Menschen eine Grenze. Hippokrates jedoch erklärt die Epilepsie zu einer körperlichen Krankheit des Gehirns und bricht mit dem religiösen Verständnis von Medizin, das Gesundheit und Krankheit vom Eingreifen der Götter abhängig macht. Die Entstehung von Krankheit definiert er vor allem als Disharmonie der vier Körpersäfte: Blut (assoziiert mit dem Herz), Schleim (Gehirn), schwarzer (Milz) und gelber Galle (Leber). Aber erst der philosophische Arzt, der zugleich in der Heilkunde und der Philosophie bewandert ist, wird für Hippokrates zum „wahren Arzt“, zum gottgleichen Iatros Philosophicus. Ungefähr zur gleichen Zeit entwickelte Demokrit eine Analogie, die das Verhältnis von Medizin und Philosophie bis ins 18. Jahrhundert prägen sollte: „Arzneikunst heilt des Leibes Krankheiten, die Weisheit befreit die Seele von den Leidenschaften“ (Demokrit 1989, S. 98).

Dieser äußerst wirkmächtige Satz wird im weiteren Verlauf von etlichen Philosophen aufgegriffen, zu metaphorischen Feldern ausgebaut und zum Topos von der Philosophie als Medizin der Seele verfestigt. An Platon kann man gut zeigen, wie Demokrits Analogie zu einem metaphorischen Feld ausgebaut wird und dadurch sehr konkrete Auswirkungen auf seine Theoriebildung hat. Platons Ideenlehre formuliert das homerische Konzept von affektgesteuerter Krankheit und vernunftgesteuerter Gesundheit der Seele aus. Vollkommene Gesundheit wird als ideales Urbild definiert, das in der unkörperlichen, völlig vernünftigen Form der Seele besteht. Erst an der verkörperten, affektgesteuerten Seele können überhaupt Krankheiten auftreten (Platon 1980, Phaid., 83d-84b; Tim., 86e). 
Ausgehend von diesem Konzept entwickelt er seine Ethik konsequent als Therapie der Seele. Weil er die Seelenkrankheiten in Analogie zur Mischung der Körpersäfte konzipiert, sei es das Ziel der Ethik, die unterschiedlichen Teile der Seele in eine naturgemäße Ordnung zu versetzen und damit gesund $\mathrm{zu}$ machen (Polit., 438d441c, 443c-445e). Entsprechend bezeichnet Platon die logischen Argumente, die Logoi, mit denen diese Harmonie hergestellt werden soll, als Medizin des Philosophen. Mit dieser logischen Medizin versucht er, die sogenannten Seelenkrankheiten seiner Schüler im Dialog zu heilen (Erler 2004).

Die Darstellung der Philosophie als Medizin der Seele hilft Platon, den abstrakten Gegenstandsbereich der Seele zu strukturieren und Handlungsanweisungen daraus abzuleiten. Außerdem dient sie als Argument gegen die konkurrierenden Rhetoren. Diese werfen den Philosophen vor, praxisferne und unnütze Reflexion zu betreiben. Wenn der Philosoph aber als Seelenarzt auftritt, logische Argumente als Medizin gelten und die Vernunft unmittelbar mit Gesundheit verbunden ist, hat er sehr gute Argumente für den pragmatischen Nutzen seiner Lehren.

Die platonische Rhetorik der gesunden Vernunft wird vor allem von den Epikureern und Stoikern aufgegriffen und $\mathrm{zu}$ einem autorenübergreifenden Topos weiterentwickelt. Der Epikureer Philodemus beschreibt das Verhältnis des Schülers zum philosophischen Lehrer in Analogie zum Verhältnis des Patienten zum Arzt. Die Argumente des Lehrers werden - wie schon bei Platon - als Medizin bezeichnet. Philodemus unterscheidet dabei aber zwischen einer milden und einer bitteren Medizin, je nach Schärfe der philosophischen Zurechtweisung. Sieht der Schüler trotz dieser Behandlungen seine Krankheiten nicht ein, kann das Gespräch aber auch in der Art einer „chirurgischen Operation“ durchgeführt werden (Philodemus 1998, S. 117).

Das ist von zweifacher Bedeutung: Einerseits hatte Aristoteles die Analogie der Arzt/Patient-Beziehung für das Verhältnis von Lehrer und Schüler noch abgelehnt, weil sie eine asymmetrische Machtbeziehung ist, in der der Patient blindes Vertrauen in die Heilmethoden des Arztes haben muss (Aristoteles 1962). Aristoteles wollte seinen Schülern in der Akademie aber auf Augenhöhe begegnen und betrachtete sie nicht als blind vertrauende Anhänger. Andererseits wurde der Vergleich zum Brennen und Schneiden der Chirurgen in der Philosophie bis zu diesem Punkt nur im Sinne der Abgrenzung benutzt. Der Philosoph sei gegenüber dem Chirurgen dadurch ausgezeichnet, dass er die ungleich sanftere Heilmethode des Gesprächs anwendete. Dass jetzt bei Philodemus nicht nur die Arzt-PatientBeziehung, sondern sogar die Chirurgie als positive Analogien zur Philosophie herangezogen werden, unterstreicht die Forderung der Epikureer nach einer direkten pragmatischen und therapeutischen Wirksamkeit ihrer Ethik. 
Die medizinischen Metaphern und Analogien werden auch von den römischen Stoikern aufgegriffen. Cicero fügt Platons Rhetorik der gesunden Vernunft einen interessanten Aspekt hinzu: Neben den Leidenschaften sind es auch falsche Urteile und Meinungen, die die prinzipiell vernünftige und gesunde Seele krank machen können: „Denn wenn die Ausgeglichenheit beim Körper, [...] Gesundheit genannt wird, so spricht man von der Gesundheit der Seele, wenn ihre Urteile und Meinungen übereinstimmen“ (Cicero 1997, S. 325-326).

Cicero verknüpft hier Gesundheit mit Kohärenz und Krankheit mit Widerspruch. Außerdem erstellt er eine Hierarchie der Krankheiten, in der die Gesund/ Krank-Polarität mit der Wahr/Falsch-Differenz zusammenfließt. Kränklichkeit und normale Krankheiten der Seele werden demnach nur als vorübergehende Widersprüche im System der Meinungen verstanden, die durch Leidenschaften ausgelöst werden. Als Steigerung der Krankheit fungiert schließlich der logische Fehler, der chronische oder unheilbare Widersprüche und Krankheiten bewirkt (Cicero 1997, S. 137-138).

\section{Christus Medicus}

Die medizinischen Topiken der antiken Philosophie werden durch das Christentum aufgenommen und modifiziert. Die Analogie von Krankheit und Leidenschaft wird prinzipiell übernommen, allerdings rückt die Sünde an die Stelle der Leidenschaften. Gott übernimmt dabei vom hippokratischen Philosophenarzt die Monopolstellung als umfassender Heiler und straft auf der anderen Seite Sünder mit Krankheit. Gottes Monopolstellung führt unweigerlich zu Konsequenzen für die weltliche Medizin. Im Zuge der Entwicklung kirchlicher Hierarchien wird der Anspruch auf ein priesterliches Monopol für Heilungen erhoben und Ärzte ohne göttliche Weihung geraten zunehmend in Verruf.

Im Neuen Testament übernimmt Jesus Christus die Rolle des Arztes. Christus Medicus - so der theologische Ausdruck - heilt Blindgeborene und wendet sich insgesamt den Kranken und Schwachen zu. War die philosophische Ethik eine Medizin der Seele, so kann das Christentum als medizinische Religion verstanden werden: „Das Christentum ist medizinische Religion: das ist seine Stärke, in manchen Ausgestaltungen auch seine Schwäche“ (Harnack 1892, S. 132). Zum Problem wurde dabei die Konkurrenz zwischen allegorischer und realistischer Interpretation, die vor allem auf die Unschärfe des christlichen Körperbegriffes zurückzuführen ist. Der Begriff Corpus Christi konnte den Leib Christi bezeichnen, aber auch metaphorisch für die Gemeinschaft der Christen oder den Textkörper der heiligen Schriften stehen. Diese drei Bedeutungsebenen werden im Folgenden etwas genauer ausgeführt. 
Die Entwicklung der Exegese des biblischen Textkörpers profitierte von Metaphern aus der hochentwickelten alexandrinischen Chirurgie. Von Origenes und Augustinus bis ins 16. Jahrhundert werden die Methoden des Zerteilens und Zergliederns des Textes von der Chirurgie entlehnt. So spricht man heute noch von der „Gliederung“ eines Textes, damals z. B. auch von dessen Skelett, oder ab dem 16. Jahrhundert sogar von seiner Anatomie (Danneberg 2003). Außerdem unterschied man einen somatischen von einem spirituellen Sinn. Während der somatische Sinn den buchstäblichen Inhalt bezeichnet, ist es das Ziel der Exegese, den allegorischen, spirituellen Sinn freizulegen (Szondi 1975).

Während sich die chirurgischen Metaphern als Gewinn für die Exegese erwiesen, entwickelte sich die Übertragung medizinischer Metaphern auf Jesus Christus vor allem für die Medizin zum Problem. Das ursprünglich allegorische Verständnis des Christus Medicus wird im 5. Jahrhundert durch die realistische Deutung der Sancti Medici, der Heiligen Heiler abgelöst. Die asketischen Sancti Medici treten jetzt in offene Konkurrenz zu den weltlichen Ärzten und verdrängen sie weitgehend. Auch die medizinischen Schriften vollziehen ab dem 5. Jahrhundert eine Abkehr von der griechischen Viersäftelehre und eine Hinwendung zu magischen Heilverfahren und Klostermedizin (Lutterbach 1996). Hier muss außerdem Erwähnung finden, dass die Kirche ihre Machtposition, die aus dem realistisch interpretierten Monopol auf Heilung resultierte, für politische Zwecke instrumentalisierte. Das zeigt sich vor allem an der Übertragung medizinischer Metaphern auf den politischen Körper der christlichen Gemeinschaft.

Als sich die Katharer im 12. Jahrhundert gegen die hierarchischen Institutionen der katholischen Kirche auflehnen, wurden sie in der Metaphorik der Seuchenbekämpfung stigmatisiert (Moore 1976). Damit wurde die Lepra zum bevorzugten Bildspender für christliche Krankheitsmetaphern. Die unterschiedlichen Krankheiten, die damals unter dem Begriff „Lepra“ zusammengefasst wurden, reichen von der Beulenpest bis hin zur Syphilis, die durch ihre sexuelle Übertragung geradezu sinnbildlich für die krankmachende Sünde steht. Das gemeinsame Merkmal dieser Krankheiten ist ihre hohe Ansteckungsgefahr, die die Kirchenväter ebenso für die häretischen Lehren der Katharer befürchteten. Im Zuge der metaphorischen Übertragung der Lepra auf die unchristlichen Lehren und Gelehrten, wurden allerdings auch die ganz unmetaphorischen Maßnahmen gegen Leprakranke auf sie angewendet. So wurden ihre Schriften verbrannt und in den außerhalb der Städte errichteten Leprosenhäusern fanden sich neben tatsächlich Erkrankten auch Häretiker, Homosexuelle und Juden wieder. Michel Foucault wird im 20. Jahrhundert in diesen Praktiken den Beginn der modernen Disziplinargesellschaft erkennen (Foucault 1969). 


\section{Gottes Apotheke und der Maschinenmensch}

$\mathrm{Zu}$ Beginn der Neuzeit inspirieren zwei gegensätzliche metaphorische Felder die entstehenden Wissenschaften: das Bild des Organischen und das des Mechanischen. Der Alchimist Paracelsus entwirft ein organistisches Weltbild, in dem die ganze Natur als eine von Gott eingerichtete Apotheke aufgefasst wird (Pagel 1962). Der Arzt hat darin die Aufgabe, die von Gott gestifteten Signaturen zu erkennen, die von den Krankheitssymptomen zu deren Medizin führen (Bergengruen 2007). Damit inspiriert Paracelsus vor allem idealistische und empiristische Tendenzen in den entstehenden Wissenschaften. Sein organistisches Modell der ,Lebenskraft‘ bleibt bis ins 18. Jahrhundert wirksam und wird für Heilmethoden wichtig, in denen Gesundheit durch eine gezielte Reizung der Lebenskraft hergestellt werden soll. Diese Methoden, die später großen Einfluss auf die Philosophie Nietzsches haben, könnte man heute am ehesten als Reizung der Abwehrkraft oder des Immunsystems verstehen.

Parallel dazu entwickeln mechanistische Metaphern große Wirkmacht vor allem auf die empiristische und rationalistische Theoriebildung. Sie kulminieren in Descartes' Modell des Maschinen-Menschen. Im Gegensatz zur Reizung der Lebenskraft artikuliert sich hier ein Konzept der Heilung, in dem der Krankheit ein kausaler Mechanismus entgegengesetzt wird. In Descartes' methodischen Zweifel kündigt sich zudem die erkenntnistheoretische Relevanz des Wahnsinns an, die er als Negativfolie des Vernunftbegriffs endgültig bei Kant erhalten wird (Derrida 1997 [1964]).

\section{Die Kritik des gesunden Verstandes}

In seiner vorkritischen Phase versucht Kant eine Ethik zu entwickeln, mit der man verbindliche moralische Vorschriften direkt aus der Vernunft ableiten kann. Er stellt sich also das Problem, einen Übergang von der Vernunft zur empirischen Welt des Handelns zu schaffen. Allerdings kann er mit keinem der konkurrierenden Vernunftbegriffe des Empirismus, Rationalismus oder Idealismus eine überzeugende Lösung für dieses Problem finden: Der Empirismus geht vom Weltlichen aus und kann von dort die geistige Sphäre moralischen Denkens nicht erfassen, Rationalismus und Idealismus gehen zwar vom Geistigen aus, schaffen es aber nicht, in die Welt menschlichen Handelns vorzudringen. Deshalb sieht Kant die Notwendigkeit, sich zunächst den Vernunftbegriffen zu widmen, bevor er sich der Ethik annehmen kann. 
Interessanterweise kündigt er seine Vernunftkritik ca. 20 Jahre vor ihrer Veröffentlichung noch als „Kritik und Vorschrift des gesunden Verstandes“ an (Kant 1977a, S. 914). Aber was genau meint er mit der ,Vorschrift des gesunden Verstandes' und warum findet sich in der Kritik der reinen Vernunft kaum ein Wort mehr über Gesundheit?

In seinen frühen Texten, dem Versuch über die Krankheiten des Kopfes (1764) und in Die Träume eines Geistersehers, erläutert durch die Träume der Metaphysik (1766), stellt Kant verwundert fest, dass die Projektionstypen psychischer Störungen verblüffende Ähnlichkeit mit den Erkenntnisprinzipien von Rationalismus, Empirismus und Idealismus aufweisen (Rauer 2007). Gerade der Übergang von der Rationalität zur empirischen Welt, der Kant für seine Ethik interessiert, läuft in jeder der drei Erkenntnistheorien Gefahr, nur ein ,Blendwerk', eine Projektion des Subjekts zu sein (Kant 1977c, S. 894). Der Empirismus ist demnach besonders anfällig für Projektionen vom Subjekt auf das Objekt, welche der Theorie der Halluzination (bei Kant: ,Verrückung`) entsprechen. Der Idealismus ist anfällig für Projektionen vom Subjekt auf das Subjekt, welche der Theorie der Bewusstseinsspaltung (,Wahnwitz') entsprechen und der Rationalismus ist anfällig für Projektionen vom Subjekt auf Subjekt und Objekt, die der Theorie der Paranoia (,Wahnsinn') entsprechen (Rauer 2007, S. 19). Hier kommt erstmalig in der Geschichte der Philosophie der Gedanke auf, die Vernunft selbst könne krank sein. Zumindest in ihrer damaligen historischen Form.

Kant folgt nun dem mechanistischen Modell der Heilung, diese Krankheiten der Vernunft mit Hilfe von Gegenmechanismen zu bekämpfen. Deshalb entwirft er drei Grundbegriffe der Kritik der reinen Vernunft - Amphibolie, Paralogismus und Antinomie - als exakte Negationen der drei psychologischen Projektionsmechanismen. Mit diesen Begriffen übersetzt Kant die psychologischen Subjekt-Objekt Relationen in grammatische und installiert einen präventiven Gegenmechanismus gegen die Anfälligkeit des Denkens für wahnhafte Projektionen. Damit vermittelt er nicht nur die unterschiedlichen Positionen in der Erkenntnistheorie, sondern versucht auch, die Vernunft bereits in ihren Grundbegriffen gegen den Wahnsinn zu immunisieren.

Es ist dabei kein Zufall, dass Kant seine Kritik im Zeitalter der Hygiene an die ,reine“ Vernunft richtet. Kant wendet sich von der psychologischen Beschäftigung mit den Geisteskrankheiten ab, weil eine Erkenntnis des pathologischen Unbewussten, wenn überhaupt, nur über Symbole vermittelt möglich ist. Es sind aber gerade die pathologischen ,Symbole“ und ,vergesellschafteten Begriffe‘, die den gesunden Verstand affizieren und psychische Krankheiten im buchstäblichen Sinn metaphorisch übertragen. Er glaubt also, dass die Ansteckungsgefahr des Wahnsinns von den symbolischen Repräsentationen des pathologisch organisierten Unbewussten, den „dunklen Vorstellungen“ ausgeht (Kant 1977b, S. 948). 
Deshalb muss die Sprache der Vernunftkritik von allen medizinischen Metaphern und Heuristiken seiner vorkritischen Schriften gereinigt werden. Und genau aus diesem Grund ist auch in der Vernunftkritik keine Rede mehr von der Gesundheit des Verstandes.

Erst auf der Grundlage dieser Vernunftkritik kann Kant sich wieder seinem ethischen Grundproblem widmen und mit dem kategorischen Imperativ eine allgemein verbindliche Regel für das Handeln aus der Vernunft ableiten. In der Kritik der praktischen Vernunft unterscheidet er außerdem zwischen dem „moralischen Gefühl“, das ebenfalls aus der Vernunft abgeleitet ist, und dem ,pathologischen Gefühl“, welches aus körperlichen Affekten hervorgeht. Mit der Ersetzung des griechischen Wortes pathos, welches gleichzeitig Krankheit und Leidenschaft bedeuten konnte, durch den relativ neuen medizinischen Ausdruck pathologisch, aktualisiert Kant den antiken Topos von der gesunden Vernunft und den krankmachenden Leidenschaften.

Kants doppelte Figur der metaphorischen Übertragung von psychischen Krankheiten und der mathematisch-modellhaften Übersetzung dieser Diagnose in eine Sprache der reinen Vernunft hat eine doppelte Konsequenz für die moderne Philosophie. Neben dem Begriff der pathologischen Projektionen wird auch die Metapher als Medium der Übertragung zu einem erkenntnistheoretischen - sogar ontologischen - Problem (Strub 1991). Mit seiner apriorischen Ableitung der Kategorien Raum und Zeit als Bedingungen der Möglichkeit von Erfahrung schafft Kant einerseits die Basis eines ,gesunden Realitätsverständnisses‘, das bis in aktuelle Definitionen allgemeiner Psychopathologie von K. Jaspers und G. Huber die theoretische Grundlage der Psychiatrie bildet (Schäfer 2006). Andererseits skizziert er auch den Problemhorizont, vor dem sich erste Ansätze zur Herausbildung einer sprachskeptischen Ontologie entwickeln, die schließlich im modernen Begriff der Metapher als „kalkuliertem Kategorienfehler“ münden sollten (Strub 1991, S. 488-489).

\section{Die Krankheit der modernen Vernunft}

Etwa hundert Jahre später verfolgt Nietzsche einen vollkommen konträren Ansatz: Anstatt jeden Anthropomorphismus zu vermeiden, tritt Nietzsche an, „sich der Analogie des Menschen zu Ende (zu) bedienen“ (Nietzsche 1999b, S. 563). Nietzsche setzt sich intensiv mit den medizinischen Theorien seiner Zeit auseinander. Er nimmt zur Kenntnis, dass sich in der zweiten Hälfte des 19. Jahrhunderts ein Bruch in der Geschichte des Krankheitsbegriffs vollzogen hat, der ,Krankheit‘ nicht mehr qualitativ, sondern nur noch quantitativ von ,Gesundheit" unterschiedet. Krankheit wird damit zu einem normalen Prozess des Lebendigen. Ge- 
sundheit ist kein idealer Urzustand mehr, sondern nur durch die Überwindung von Krankheiten erreichbar. Darüber hinaus ist Nietzsche fasziniert vom physiologischen Prozessdenken, den elektrischen Übertragungen des Nervensystems, dem Stoffwechsel und dem Blutkreislauf. In diesen damals neuen physiologischen Entdeckungen offenbaren sich Prozesse, deren zirkuläre Ökonomien Kants scheinbar so klare Gegenüberstellung von Vernunft und Krankheit durchkreuzen. Nach seinen medizinischen Recherchen konzipiert Nietzsche die Entstehung der Sprache als neurophysiologische Übertragung eines „Nervenreizes“ in ein „Bild“ und einen „Laut“ (Nietzsche 1999a, S. 879). Das Ergebnis dieser Übertragungen ist jeweils eine Metapher, die wie der Nervenreiz ohne eine darunterliegende Logik von einer Sphäre zur nächsten springt. Das Überspringen der Kategorien vom Denken zum Handeln, oder vom Körper zum Text, das Kant noch als Kontagium der Sprache deklariert hatte, wird hier zu ihrem physiologischen Grundprinzip erklärt.

Das Konzept des Leibes verschmilzt so mit dem Konzept der Sprache bis zur Ununterscheidbarkeit; körperliche organische Prozesse der Selbstregulierung müssen gleich als „Interpretationen“ sprachlich gefasst werden (Nietzsche 1999c, S. 139-140). Jede Bewegung des Organischen, also auch das menschliche Verhalten und das Denken sind aus dieser Perspektive betrachtet „Symptome“ und „Zeichen“ eines psycho-physischen Geschehens (Nietzsche 1999c, S. 16-17). Diese Symptome können nicht mehr kategorial unter Gesund/Krank-, Wahr/ Falsch-, Begriff/Metapher-Unterscheidungen subsumiert werden, sondern müssen im Prozess zwischen den jeweiligen Kristallisationen gedacht werden. Erst das Erstarren der Metapher im konventionellen Begriff beendet den ,lebendigen“ Akt des schöpferischen Sprachkörpers und seiner literalen Körpersprache.

Die „Krankheit der Sprache“ und damit der modernen Vernunft besteht für Nietzsche deswegen im „Wahnsinn der allgemeinen Begriffe“ (Nietzsche 1999e, S. 455). Die Definition eines Begriffes stoppt demnach den lebendigen Prozess der Interpretation, den Nietzsche auch als ,Einverleibung' bezeichnet. Bis in seine späten Schriften ist der Versuch erkennbar, die feststellenden Begriffsgebäude der Philosophie metaphorisch zu verflüssigen und medizinische Denkfiguren immer detaillierter zu präzisieren. Dabei erhält der Philosoph die Aufgabe, als „Arzt der Kultur“ aufzutreten und zur Überwindung der von Nietzsche diagnostizierten historischen und moralischen Krankheiten beizutragen. Gleich in seiner ersten philosophischen Diagnose der „historischen Krankheit“ rechnet er mit der akademischen Philologie ab und verschreibt der vom Historismus dominierten Philosophie einen medizinischen Perspektivwechsel: eine „Gesundheitslehre des Lebens“ soll „neben die Wissenschaften“ gestellt werden (Nietzsche 1999e, S. 329).

Bevor sich diese Diagnose im 20. Jahrhundert auf zwei völlig unterschiedliche Weisen realisieren sollte, von denen sich in letzter Konsequenz weder die Psy- 
choanalyse noch die Rassenhygiene sinnvoll auf Nietzsche berufen kann, bastelt Nietzsche am neuen Typus eines modern-antiken Philosophenarztes, der die „Symptome‘ seiner jeweiligen Gegenwart ,diagnostiziert' und philosophische ,Therapien', ,Stimulanzen` und ,Reizmittel' an diese Diagnosen anpassen soll (Pasely 1978).

Für Nietzsche erfüllen alle „morbiden Zustände“ die Funktion eines „Vergrößerungsglas[es]“ für Phänomene, „die normal aber als normal schlecht sichtbar sind“ (Nietzsche 1999d, S. 250). Dieselbe Funktion kann der Metapher auf sprachlicher Ebene zugesprochen werden, weil sie mit dem innovativen Effekt des Perspektivwechsels kategorienübergreifende Einsichten unterstützt und Kontexte variiert. Damit wird Kants Einschätzung der Metapher als Medium der Übertragung psychischer Verrückung und Verwirrung unter umgekehrten Vorzeichen wiederholt. Während Kant die Sprache im Paradigma der Hygiene von ihren potentiell kontagiösen Elementen kategorialer Verrückung mit Gegenmechanismen $\mathrm{zu}$ reinigen versucht, setzt Nietzsche kalkuliert dieselben Elemente als Stimulanzien gegen eine Vernunftkonzeption ein, deren mathematischer Präzisionswille das Leben zu lähmen droht.

\section{Vernunft als Therapie und Krankheit: Kritische Reflexionen}

Dieser stark verkürzende historische Abriss sollte verdeutlichen, dass sich die Vernunftbegriffe der europäischen Philosophie seit der Antike in einem unterschwelligen Verweisungszusammenhang auf die Gesund/Krank-Differenz entwickelten. Die antike Analogie von der Philosophie als Medizin der Seele wurde zu ganzen Feldern medizinischer Metaphern ausgebaut und als autorenübergreifender Topos tradiert. Anhand der Akzeptanz oder Ablehnung bestimmter Metaphern und Analogien ließ sich sogar rekonstruieren, ob ihre Verwendung in erster Linie auf Erkenntnisgewinn, oder auf Machtakkumulation und -erhaltung abzielte. Vor allem die chirurgischen Metaphern, die seit den Epikureern vermehrt Verwendung fanden, erwiesen sich als anfällig für paternalistische und stigmatisierende Effekte.

Mit der Entstehung der Psychologie und ihrer Beschäftigung mit dem Wahnsinn wurde die Rhetorik der gesunden Vernunft durch das Aufkommen der Metapher einer kranken Vernunft erschüttert. Sie wird zunächst bei Kant implizit heuristisch genutzt, dann bei Nietzsche als prozessuales Moment der gegenseitigen Durchdringung von Leib und Sprache expliziert. Beide Autoren beziehen sich dabei auf unterschiedliche Modelle der Heilung. Kant setzt im Zeitalter der 
Hygiene auf ein mechanistisches Modell, das der diagnostizierten Krankheit der Vernunft einen begrifflichen Gegenmechanismus entgegensetzt. Das Kant die AntiProjektions-Mechanismen in die allgemeinen Begriffe der Vernunft einbringt, geht mit einem gewissen Paternalismus und einem passiven ,Patientenverhalten“ einher. Denn alle Menschen, welche die Vernunft in dieser Form gebrauchen, sollen ohne weiteres Zutun gegen den Wahnsinn immun sein. Je weniger sie von diesen Mechanismen wissen, desto besser sind sie sogar immunisiert.

Im Zeitalter der Nervosität löst Nietzsche die starre Gegenüberstellung von Vernunft und Krankheit neurophysiologisch auf und versucht, dem organistischen Modell der Reizung der Lebenskraft folgend, die ,große Vernunft des Leibes“ durch metaphorische Irritationen zu stimulieren und zu steigern. Im Gegensatz zu Kants Paternalismus der allgemeinen Begriffe setzt Nietzsche vielmehr auf die individuelle Antwort auf eine Krankheit. Er fordert eine aktive Interpretation und Antwort des ,Patienten“ auf die ,Krankheit‘. Sein Denken weist eher in die Richtung eines salutogenetischen Gesundheitsverständnisses, welches auf das ,Coping` abzielt, also auf die individuelle Fähigkeit des Organismus, auf Krankheiten zu reagieren.

Von beiden Autoren lassen sich Traditionslinien in die jüngere Geschichte der Philosophie zeichnen, die auch hinsichtlich ihrer Verständnisse von Gesundheit und Modelle von Heilung konsistent erscheinen. Von Nietzsche führt eine Linie über Sigmund Freud und Ludwig Wittgenstein zu den Autoren der französischen Postmoderne und der Systemtheorie. Derrida setzt Wittgensteins „Selbstheilung philosophischer Denkkrankheiten“ fort (Nohr 2015, S. $303 \mathrm{ff}$.), indem er seine Philosophiekritik in den Diagnosen des Anthropo-, Logo- und Ethnozentrismus artikuliert und mit der Affirmation viraler und psychoanalytischer Metaphern begegnet. Damit soll der logozentristische Wahrheitsdiskus irritiert werden, indem der Unterschied von Begriff und Metapher insgesamt in Frage gestellt wird.

Von Kant führt eine andere Linie über Karl Jaspers und Viktor v. Weizsäcker zu Jürgen Habermas. Habermas’ Diskursethik setzt den kranken Kontexten einer „kapitalistisch überformten“ und „kolonialisierten Lebenswelt“ die therapeutische Wirksamkeit der „idealen Sprechersituation“ und ihrer Begriffssprache entgegen (Habermas 1981, S. 539 ff.).

Neben diesen impliziten, oft versteckten, metapherngeschichtlichen Importen medizinischer Denkfiguren in die Philosophie, bezeugt die klassische Begriffsgeschichte den philosophischen Begriffsexport. So bildeten sich allein in der zweiten Hälfte des 20. Jahrhunderts eine Vielzahl theoretischer Ansätze heraus, die funktionalistische, normative und systemtheoretische Krankheitsbegriffe umfassen und sozialwissenschaftliche, psychosomatische sowie salutogenetische Forschungen beinhalten. Die im Nachkriegseuropa vor allem von Georges Canguilhem, Karl Jaspers und Ivan Illich, in den USA von Christopher Boorse, 
Talcott Parsons und Aaron Antonovsky geführte philosophische Diskussion um den Krankheitsbegriff behandelte im Wesentlichen Themen der Subjektivität/ Objektivität und Normativität/Deskriptivität des Krankheitsbegriffs sowie die Relationierung seiner somatischen, psychischen und sozialen Dimensionen (Lanzerath 2000). Es scheint, dass sogar die Erkenntnistheorien der Moderne nicht ohne Krankheitsmetaphern auskommen und ihre Rationalitätskonzepte erst vor dem Hintergrund der zeitgenössischen Medizindiskurse verständlich werden.

Jenseits der Arbeit am Begriff ist die Metapher in den letzten Jahrzehnten immer stärker in den Fokus philosophischer Aufmerksamkeit gerückt, wobei sowohl Metapherntheorien (Rolf 2011) als auch Metapherngeschichten (Kroß \& Zill 2011) eine Konjunktur verzeichnen konnten. Betrachtet man vor diesem Hintergrund die jüngsten Versuche von Medizinern und Philosophen, eine sowohl praktikable, theoretisch konsistente und ethisch vertretbare Definition von Krankheit zu erarbeiten, wird die Differenz von Krankheitsbegriff und -metapher insgesamt problematisch. Der aktuell gebräuchliche, systemtheoretische Krankheitsbegriff bezieht sich beispielsweise gar nicht mehr auf Lebewesen, sondern auf die Kopplung bio-psycho-sozialer Systeme (Uexküll \& Wesiack 1991). Die Bindestriche in diesem Begriff übernehmen dabei gewissermaßen die Funktion der Metapher: den Sprung über Kategoriengrenzen.

Wenn Metaphern in den Wissenschaften grundsätzlich keinen guten Ruf genießen, gilt dies insbesondere für Krankheitsmetaphern (Sontag 2003). Zuletzt ist ihre Bedeutung für die pseudo-medizinische Legitimation und Durchführung des Holocaust umfangreich erforscht worden (Sarasin 2007). Mit dem Paradigmenwechsel von der Hygiene zur Bakteriologie wurden Krankheiten demnach nicht mehr auf äußere Umstände, sondern auf labortechnisch identifizierbare Pathogene zurückgeführt. Diese medizinische Visualisierung des Feindes, welche Robert Koch durch die Markierung von Gewebeschnitten mit Anilinfarbe entscheidend voranbrachte, bot zusammen mit der monokausalen Erklärung von Krankheiten große metaphorische Anschlussflächen für die politischen Diskurse, die sich seit dem 19. Jahrhundert mehr und mehr mit der „Sicherung des Lebens“ beschäftigten (Sarasin 2007, S. 427-428). Dabei ergänzte und befeuerte sich die politische Metaphorik der Mediziner mit der medizinischen Metaphorik der Politiker. Während Koch die „Einwanderung der Bazillen“ in den menschlichen Körper zur Invasion der „Zerstörer der Menschheit“ stilisierte (Koch 1912 [1882], S. 429), die es mit allen monetären, technischen und industriellen Mitteln der neuen Medizin zu „bekämpfen“ gelte, wurden die Begriffe ,Bakterium“ und ,Bazille“ in politischen Diskursen zunächst metaphorisch auf Migranten aus dem Osten angewendet, bis später das Bild vom Juden als ,Bazille im arischen Volkskörper‘ zum Instrument faschistischer Propaganda avancierte. 
Stärker noch als im so genannten ,Sozialdarwinismus', in welchem der Kampf zwischen den Rassen und Kulturen im Hinblick auf eine Vervollkommnung und Verbesserung für die Zukunft perspektiviert wurde, zielt die Bildsprache der Bakteriologie auf eine vollkommen apokalyptische Form gegenseitiger Auslöschung, deren national-sozialistische Durchführung nicht zufällig mit der Sichtbarmachung der Juden per gelbem Davidstern beginnen sollte (Broszat 1977).

Aber nicht erst die Geschichte des Holocaust belegt, dass gerade ontologisierende Krankheitsbegriffe wie der bakteriologische sich als effektives Mittel erweisen, politische Gegner bis zur Ermordung zu verfolgen. Von den christlichen Glaubens-kämpfen und dem amerikanischen Bürgerkrieg (Cartwright 1851) über den Genozid der Türken an den Armeniern von 1916 (Kieser \& Schaller 2002) bis zu den sogenannten Säuberungswellen in der Sowjetunion (Weiss 2005) führt die Spur medizinischer Metaphern in den biopolitischen Legitimationsdiskursen zum Genozid.

Vor dem Hintergrund politisch-medizinischer Metaphorik zeigt ein Blick auf die COVID-19-Pandemie, dass Krankheiten immer noch mit Kriegsrhetorik beschrieben werden. Emmanuel Macron wiederholte den Satz: „Wir sind im Krieg“ in seiner Rede an die Nation gleich sechs Mal. Donald Trump bezeichnete sich als „Kriegspräsident“ und rief auf seiner täglichen Pressekonferenz erneut den „Kampf“ gegen einen „unsichtbaren Feind“ aus, während sich die Ärztinnen und Ärzte im italienischen Bergamo als „Soldatinnen“ an der „,vordersten Front“ begriffen. So scheint es, als könnten durch die Jahrhunderte hinweg weder Philosophie noch Politik der Versuchung widerstehen, an medizinische Diskurse anzuschließen. Dies mag daran liegen, dass Gesundheit ein derart verbindendes Element ist: Die Notwendigkeit für Gesundheit erschließt sich jedem Menschen unmittelbar. In dieser unmittelbaren Notwendigkeit liegt jedoch nicht nur die große Anziehungskraft medizinischer Denkfiguren begründet, sondern auch ihr potentieller Missbrauch. Es bleibt zu hoffen, dass sich insbesondere die Politik hier ihrer Verantwortung bewusst ist.

\section{Literatur}

Aristoteles (1962): „Eudemische Ethik“. In: Werke in deutscher Übersetzung, Bd. 7 (Ernst Grumach und Hellmut Flashar, Hrsg.). Berlin: Akademie-Verlag.

Bergengruen, Maximilian (2007): Nachfolge Christi - Nachahmung der Natur. Hamburg: Meiner.

Broszat, Martin (1977): „Hitler und die Genesis der Endlösung“. In: Vierteljahrshefte für Zeitgeschichte 25, S. 739-775.

Cartwright, Samuel (1851): „Report of Diseases and Physical Peculiarities of the Negro Race“. In: The New Orleans Medical Journal May 1851, S. 318-325. 
Cicero (1997): Tusculanae disputationes = Gespräche in Tusculum, Bd. 4. Stuttgart: Reclam.

Danneberg, Lutz (2003): Anatomie des Text-Körpers und des Natur-Körpers. Berlin \& New York: De Gruyter.

Demokrit (1989): Demokrit. Texte zu seiner Philosophie (Rudolf Löbl, Hrsg.). Amsterdam: Rodopi.

Derrida, Jacques (1997 [1964]): „Cogito und die Geschichte des Wahnsinns“. In: Jaques Derrida (Hrsg.): Die Schrift und die Differenz. Frankfurt am Main: Suhrkamp, S. 53-101.

Erler, Michael (2004): „Sokrates in der Höhle - Argumente als Affekttherapie im ,Gorgias“ und im ,Phaidon““. In: M. v. Ackeren (Hrsg.): Platon verstehen. Darmstadt: wbg Academic, S. $57-68$.

Foucault, Michel (1969): Wahnsinn und Gesellschaft. Frankfurt am Main: Suhrkamp.

Habermas, Jürgen (1981): Theorie des kommunikativen Handelns. Frankfurt am Main: Suhrkamp.

Harnack, Alfred von (1892): Medicinisches aus der ältesten Kirchengeschichte. Leipzig: Hinrichs.

Homer (2002): Ilias. München: dtv.

Kant, Immanuel (1977a): „Nachricht von der Einrichtung seiner Vorlesungen in den Winterhalbjahren von 1765-66“. In: Werkausgabe in 12 Bänden, Bd. 2.2 (Wilhelm Weischedel, Hrsg.). Frankfurt am Main: Suhrkamp, S. 907-914.

Kant, Immanuel (1977b): „Träume eines Geistersehers, erläutert durch die Träume der Metaphysik“. In: Werkausgabe in 12 Bänden, Bd. 2.2 (Wilhelm Weischedel, Hrsg.). Frankfurt am Main: Suhrkamp, S. 923-989.

Kant, Immanuel (1977c): „Versuch über die Krankheiten des Kopfes“. In: Werkausgabe in 12 Bänden, Bd. 2.2 (Wilhelm Weischedel, Hrsg.). Frankfurt am Main: Suhrkamp,

S. $887-901$.

Kieser, Hans-Lukas/Schaller, Dominik (Hrsg.) (2002): Der Völkermord an den Armeniern und die Shoa/The Armenian Genocide and the Shoa. Zürich: Chronos.

Koch, Robert (1912 [1882]): „Die Ätiologie der Tuberkulose“. In: Gesammelte Werke, Bd. 1 (J. Schwalbe, Hrsg.). Leipzig: Georg Thieme, S. 428-444.

Kroß, Matthias/Zill, Rüdiger (Hrsg.) (2011): Metapherngeschichten. Perspektiven einer Theorie der Unbegrifflichkeit. Berlin: Parerga.

Lanzerath, Dirk (2000): Krankheit und ärztliches Handeln. Zur Funktion des Krankheitsbegriffs in der medizinischen Ethik. Freiburg \& München: Karl Alber.

Lutterbach, Hubertus (1996): „Der Christus medicus und die Sancti medici“. In: Saeculum 47, S. $239-281$.

Moore, Richard Irving (1976): „Heresy As Disease“. In: Willem Lourdaux/Daniël Verhelst (Hrsg.): The Concept Of Heresy In Middle Ages (11th-13th c.). Leuven: University Press, S. $1-11$.

Nietzsche, Friedrich (1999a): „Nachgelassene Fragmente 1869-1874“. In: Sämtliche Werke. Kritische Studienausgabe in 15 Bänden, Bd. 7 (Giorgio Colli/Mazzino Montinari, Hrsg.). München: De Gruyter.

Nietzsche, Friedrich (1999b): „Nachgelassene Fragmente 1884-1885“. In: Sämtliche Werke. Kritische Studienausgabe in 15 Bänden, Bd. 11 (Giorgio Colli/Mazzino Montinari, Hrsg.). München: De Gruyter. 
Nietzsche, Friedrich (1999c): „Nachgelassene Fragmente 1885-1887“. In: Sämtliche Werke. Kritische Studienausgabe in 15 Bänden, Bd. 12 (Giorgio Colli/Mazzino Montinari, Hrsg.). München: De Gruyter.

Nietzsche, Friedrich (1999d): „Nachgelassene Fragmente 1887-1889“. In: Sämtliche Werke. Kritische Studienausgabe in 15 Bänden, Bd. 13 (Giorgio Colli/Mazzino Montinari, Hrsg.). München: De Gruyter.

Nietzsche, Friedrich (1999e): „Unzeitgemäße Betrachtungen“. In: Sämtliche Werke. Kritische Studienausgabe in 15 Bänden, Bd. 1 (Giorgio Colli/Mazzino Montinari, Hrsg.). München: De Gruyter.

Nohr, Olaf (2015): Vernunft als Therapie und Krankheit. Berlin: Logos.

Pagel, Walter (1962): Das medizinische Weltbild des Paracelsus. Wiesbaden: Franz Steiner.

Pasely, Malcom (1978): „Nietzsches Use of Medical Terms“. In: Malcom Pasely (Hrsg.): Nietzsche. Imagery and Thought. A Collection of Essays. London: Methuen, S. 123-158. Philodemus (1998): On Frank Criticism. Atlanta: Scholars Press.

Platon (1980): Sämtliche Werke (Ernesto Grassi/Walter Hess, Hrsg.). Hamburg: Rowohlt. Rauer, Christoph (2007): Wahn und Wahrheit. Berlin: Akademie-Verlag.

Rolf, Eckard (2011): Metaphertheorien. Typologie - Darstellung - Bibliographie. Berlin \& Boston: De Gruyter.

Sarasin, Phillip (2007): „Die Visualisierung des Feindes. Über metaphorische Technologien der frühen Bakteriologie“. In: Phillip Sarasin (Hrsg.): Bakteriologie und Moderne. Studien zur Biopolitik des Unsichtbaren 1870-1920. Frankfurt am Main: Suhrkamp, S. 427-461.

Schäfer, Axenia (2006): Das Kantische Subjekt in der Psychopathologie. Marburg: Tectum.

Schmitz, Hermann (1965): Der Leib. Bonn: Bouvier.

Sontag, Susan (2003): Krankheit als Metapher \& AIDS und seine Metaphern. München: Hanser.

Strub, Christian (1991): Kalkulierte Absurditäten. Versuch einer historisch reflektierten sprachanalytischen Metaphorologie. Freiburg im Breisgau: Karl Alber.

Szondi, Peter (1975): Einführung in die literarische Hermeneutik. Frankfurt am Main: Suhrkamp.

Uexküll, Thore von/Wesiack, Wolfgang (1991): Theorie der Humanmedizin. München, Wien \& Baltimore: Urban \& Schwarzenberg.

Weiss, Daniel (2005): „Ungeziefer, Aas und Müll. Feindbilder in der Sowjetpropaganda“. In: Österreichische Zeitschrift für Geschichtswissenschaften 16(3), S. 109-122. 